\title{
Damage detection by using FBGs and strain field pattern recognition techniques
}

\author{
Julián Sierra-Pérez , Alfredo Güemes and Luis E Mujica \\ ${ }^{1}$ Aerospace Engineering Research Group, Universidad Pontificia Bolivariana, Medellín, Colombia \\ ${ }^{2}$ Department of Aerospace Materials and Processes, Universidad Politécnica de Madrid, Madrid, Spain \\ ${ }^{3}$ Department of Applied Mathematics III, Universitat Politécnica de Catalunya, Barcelona, Spain
}

\begin{abstract}
A novel methodology for damage detection and location in structures is proposed. The methodology is based on strain measurements and consists in the development of strain field pattern recognition techniques. The aforementioned are based on PCA (principal component analysis) and damage indices ( $T^{2}$ and $Q$ ). We propose the use of fiber Bragg gratings (FBGs) as strain sensors.
\end{abstract}

\section{Introduction}

In many engineering fields, the integrity and reliability of structures are extremely important aspects. These are controlled by adequate knowledge of existing damage. Typically, achievement of the level of knowledge necessary to characterize the structural integrity involves the use of nondestructive testing (NDT) techniques. These are often expensive and time consuming.

Nowadays, many industries look to increase the reliability of their structures by using leading edge techniques to monitor them and, in some cases, detect incipient damage that could trigger catastrophic failure. Unfortunately, as the complexity of the structures, components and systems increases, the risk of damage and failure also increases. At the same time, the detection of such failure and defects becomes more difficult.

In recent years, the aerospace industry has made a great deal of effort to integrate sensors within structures and to develop algorithms to determine the structural integrity in real time. The philosophy is called 'structural health monitoring' and these structures are called 'smart structures'. These new types of structures integrate materials, sensors, actuators and algorithms to detect, quantify and locate damage within themselves.

Structural health monitoring (SHM) is the identification of four characteristics that are related to the physical state of an engineering component (or system) as it operates. Such identification will not affect the integrity of the structure,
First, the operational and environmental loads that interact with the component or system, second, load induced damage, third, damage growing as the component or system operates and fourth, the performance of the component or system as it accumulates damage during service are monitored. When these SHM systems are fully developed, they will reduce the time and costs associated with maintenance by eliminating unnecessary inspections and replacements (Balageas et al 2006, Adams 2007).

The basic characteristic of SHM that differentiates it from conventional nondestructive evaluation (NDE) is that sensors are permanently attached to the structure, working, therefore, for the whole operational life of the structure without requiring operator intervention. A useful system usually requires a large number of sensors distributed throughout the structure. The large amount of data produced must be automatically processed. Warning signals need to be filtered to the user when there is structural overload, damage detection, or to produce reports on the fatigue accumulated by the structure.

SHM systems currently use modern experimental techniques that allow very precise measurement of different types of structural response to external loads. The result of the measurements can be subsequently analyzed by numerical and statistical techniques to identify the onset of damage. Generally, current SHM systems consist of sensors, data acquisition and preprocessing blocks of data (software), data communication systems and post-processing blocks of data (Holnicki-Szulc 2008). 
The first works on damage detection used identification techniques based on physical systems, for example, determination of the stiffness matrix or modal parameters. These approaches deal with deterministic models where all parameters are considered as measurable and different uncertainties are not represented for the model directly. This causes difficulty in assessing how reliable the damage estimates are (Li et al 2012).

A more recent technique estimates the occurrence of damage based on experimental data. These models take experimental measurements for 'training' or learning and assessment of the current state of a component or structure. These methods are a very robust way to indicate the presence of damage.

Structural health monitoring includes two lines of action that are independent in their procedures, but complementary in their application: load monitoring or continuous measurement of strains, and damage monitoring or autonomous systems to detect irregularities or flaws in the structure.

There are several physical principles associated with the structure that can be studied as damage initiates and grows. In this work, there is particular interest the global stiffness and the strain field. Any defect will change the strain field of the structural element and its global stiffness slightly. These changes can be experimentally detected. Correlation between them and the occurrence, location, and quantification of damage is a very complex problem which is much less well resolved that monitoring loads (Fritzen and Kraemer 2009).

Many techniques are promising in damage detection. Each technique uses a particular physical principle. In general the techniques can be classified as follows(Ostachowicz 2011):

- vibration based methods,

- guided wave based methods,

- optical fiber based methods,

- acoustic emission based methods,

- differential pressure based methods,

- electromagnetic field based methods.

The construction of aircraft and space structures requires increasingly sophisticated designs and materials. This increases the complexity of physical/mathematical models that can accurately describe the relationships between actions and responses in the structures. This leads to the need to explore and develop signal processing techniques that do not require explicit use of physical/mathematical models while being efficient and robust against uncertainties. This is the basis for the methodology of pattern recognition.

An additional problem is that normally SHM systems use tens of sensors measuring different variables for long periods of time. This creates an overload of data and usually many of these data should be discarded. There is both correlatable and redundant information in the measurements. A data treatment process is necessary to allow the elimination of noise or irrelevant data and the study of the different correlations.
The main objective of this work is to develop and experimentally validate SHM tools that allow detection and location of defects in aerospace and civil structures based the approach on strain measurements and using strain field pattern recognition techniques. We propose the use of Bragg gratings for strain measurements, dimensional reduction tools and multivariate analysis like the study of principal component analysis (PCA) (Jolliffe 2002). The potential of this technique is that it does not require the use of models which, given the complexity of the structures, materials and environmental uncertainties, are difficult to apply accurately.

An innovative way to incorporate PCA in operational variable conditions (like variable loads) is developed for this particular application. This innovative adaptation of PCA to strain field pattern recognition includes the development of damage indices and detection thresholds.

\section{SHM in practice}

In general, no matter which SHM techniques are used, the steps are the same: (1) operational evaluation, (2) data acquisition, (3) standardization, cleaning, selection and condensation of information (preprocessing), (4) implementation of a model for discrimination of information and (5) situational assessment and decision making (Mujica et al 2010a, 2010b, Lopez and Sarigul-Klijn 2010).

There are many preprocessing techniques that can treat data acquisition errors, noise, transformation and compression. Many signal processing techniques include Fourier analysis for stationary time-invariant problems and time-varying methods such as wave methods (wavelets), time-frequency methods and time series analysis. The main methods for time-frequency analysis are short time Fourier transform (STFT), Wigner-Ville distribution (WVD), Choi-Williams distribution (CWD), among others. The main difference between the different time-frequency methods is the treatment of uncertainty (Lopez and Sarigul-Klijn 2010, Baseville et al 2007, Li et al 2012).

Wave techniques serve as tools for time-varying analysis and are useful for decomposition, compression and selection of features. Some of the best known wave methods include continuous, discrete and multiple resolution methods (Reda et al 2006).

Time series techniques use statistical tools to develop mathematical models that describe one or more measured stochastic signals and analyze observed and future behavior (Worden and Farrar 2007).

After data preprocessing, many techniques are used for discrimination of information whose general purpose is to find information and highlight hidden patterns in data. In this way, it is possible to manage the information for optimization purposes, decision support and control processes, among others (Staszewski 2004).

There are different techniques of multivariate statistical process control that reduce the dimensionality of sample populations. Dimensional reduction is a way of transforming the vectors $X \in R^{n}$ into new vectors $X \in R^{m}$, where $m<n$ (Westerhuis et al 1999). 


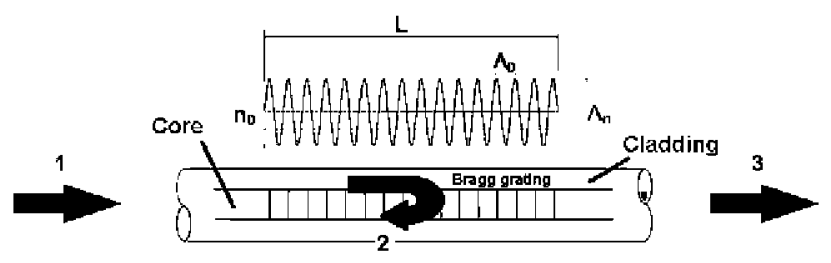

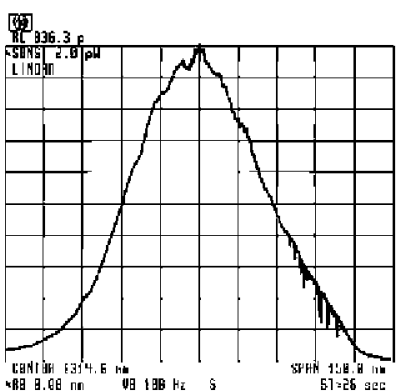

(1)

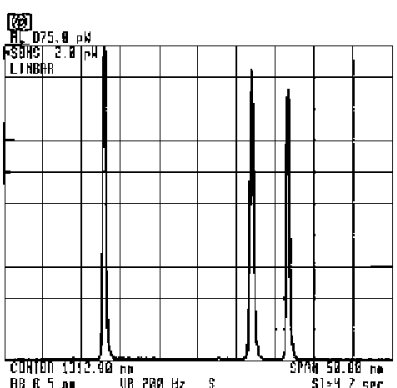

(2)

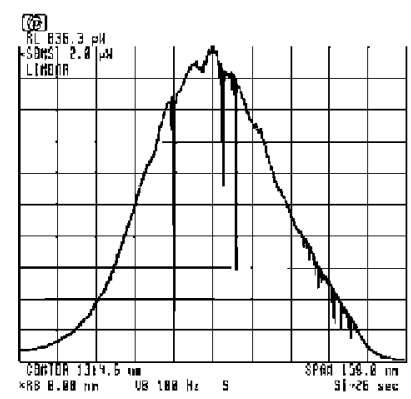

(3)

Figure 1. A fiber Bragg grating (FBG) and schemes of its operating principle. (1) The intensity spectrum of a broadband source launched into the fiber. (2) Spectra reflected back by three fiber Bragg gratings. (3) The transmissive spectrum after passing the three Bragg gratings.

The strength of the different techniques used for dimension reduction is that they are able to transform highly correlated, redundant or noisy data to a statistical model whose elements provide an overview of hidden phenomena and correlations that determine the behavior of the system (Westerhuis et al 1999, Lopez and Sarigul-Klijn 2010, Mujica et al 2006, 2008).

Some of the techniques currently available include, for example, Markov models, linear regressions, ROM (reduced order modeling), SVD (singular value decomposition), PCA (principal component analysis), also called Karhunen-Loeve expansion, multiway PCA (MPCA), also called Tucker 1, PARAFAC, Tucker 3, PLS (partial least square), etc (Westerhuis et al 1999).

The best known technique for extracting information, dimensional reduction and characteristic identification is the PCA. For this reason, it is perhaps the most widely used technique at present. The ultimate goal of the technique is to discern which data represent the most important dynamics of a particular system and which are redundant or are simply noise. This goal is achieved by determining a new space that allows re-expression the data based on the original data covariance structure (Mujica et al 2010a, 2010b).

Finally, situational assessment and decision making are carried out. In the particular case of SHM this consists in determining the structural health and the assessment of damage. The situational assessment (and classification) is the most important and most significant task in SHM development.

Many classification techniques have been proposed in SHM. Among the most important features are similar metrics, decision tree induction, Bayesian classification, neural networks, genetic algorithms, fuzzy classifiers, and more. However, at present, the most studied and best performing method is still statistical analysis (Lopez and Sarigul-Klijn 2010, Kerschen and Golinval 2004, Nair and Kiremidjian 2006, Worden and Dulieu-Barton 2004, Staszewski 1997 ,
Fugate et al 2001, Sohn et al 2000, Worden and Manson 2000).

\section{Fiber optic sensors}

Concerning fiber optic sensors, fiber Bragg gratings (FBGs) are the most widely available commercial systems today because of their high performance and comparative advantages compared to other strain measurement techniques. Some of these advantages are high sensitivity, small size that allows them to be embedded directly into composite materials, low weight, less signal degradation (immunity to electromagnetic interference and radio frequency), low power consumption, non-flammable, user friendly, moderate cost, high operating temperatures and high fatigue resistance.

FBGs are regions in an optical fiber where the core has a periodic variation of its diffraction index with a period lambda $(\Lambda)$. This modulation is induced in a special type of optical fiber (photosensitive) by exposing the core to light from an ultraviolet laser. The laser interacts with a diffraction grating (phase mask) of a determined wavelength (period) between the laser and the optical fiber. In this way, a Bragg grating with a given period and a length ranging between 1 and $20 \mathrm{~mm}$ is 'written' on the fiber optics.

When light passes through an FBG, either from a white light source or from a tunable laser, the FBG behaves as a band elimination filter, as shown in figure 1 . In each region where the refractive index increases locally, part of the incident light is reflected. These increments are caused by the periodic modulation induced in the fiber. Since these regions are equally spaced, the wavelengths correspond to integer multiples of the modulation length, will experience constructive interference and will be reflected. This phenomenon does not occur for other wavelengths.

Bragg gratings can be used as strain and temperature sensors, among others. To be employed as strain sensors, the FBGs must be adhered to or embedded in the material 


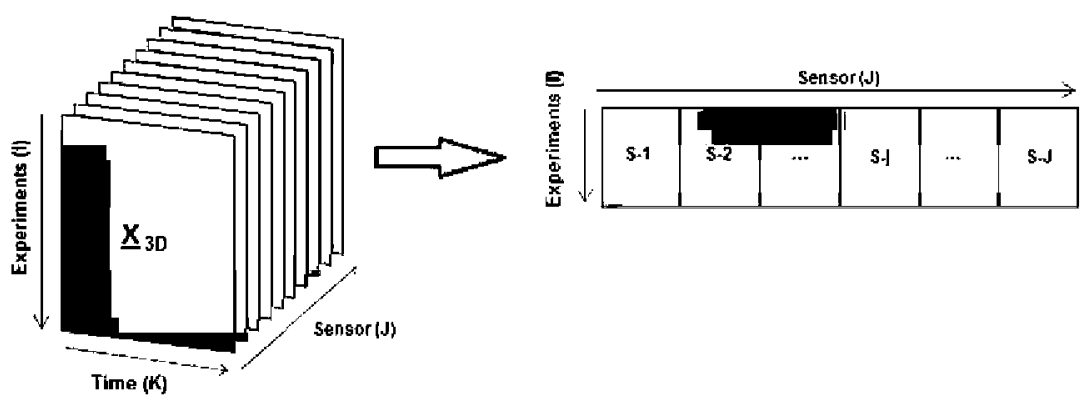

Figure 2. The 'type D' unfolding model.

in which the strain measurement is desired. Thus, the FBG will deform integrally with the substrate to which it has been bonded. As a result of deformation, the modulation period $\Lambda$ will vary. The Bragg length will vary as well. Similarly, the effective diffractive index varies with temperature so that the Bragg wavelength is also sensitive to changes in temperature.

\section{Principal component analysis}

The measurements performed in SHM techniques normally use different sensors, measuring continuous dynamic signals as a function of time. Therefore, it is necessary to perform a treatment of the experimental data prior to application of the PCA technique. In the first instance it is necessary to discretize the signals in order to obtain a manageable data set. An $\mathbf{X}$ matrix with all data information from the measurement of several variables $(m)$ for a number of experimental trials $(n)$ can be arranged as follows:

$$
\begin{aligned}
\mathbf{X} & =\left[\begin{array}{cccccc}
x_{11} & x_{12} & \cdots & x_{1 j} & \cdots & x_{1 m} \\
\vdots & \vdots & \ddots & \vdots & \ddots & \vdots \\
x_{i 1} & x_{i 2} & \cdots & x_{i j} & \cdots & x_{i m} \\
\vdots & \vdots & \ddots & \vdots & \ddots & \vdots \\
x_{n 1} & x_{n 2} & \cdots & x_{n j} & \cdots & x_{n m}
\end{array}\right] \\
& =\left(\begin{array}{llllll}
v_{1} & v_{2} & \cdots & v_{j} & \cdots & v_{m}
\end{array}\right) .
\end{aligned}
$$

Each row vector $\left(x_{i}\right)$ represents the measurements from all sensors for a specific time instant or experimental trial, each column vector $\left(v_{j}\right)$ represents the measurements from one sensor (variable) for the whole set of experiment trials.

If each variable is a sensor that measures dynamic signals, all the gathered information can be arranged as a tridimensional matrix ( $I$ experiments $\times K$ samples $\times J$ sensors); see figure 2. Each frontal slice represents all measurements from one sensor for the whole experiment. This matrix must be unfolded to perform a PCA study (Kouti and MacGregor 1995).

There are several ways to unfold 3D data arrays in the literature; each one allows the study of a different kind of variability by means of principal component analysis. According to Nomikos and MacGregor, the most used way to unfold 3D data arrays is the so-called 'D type unfolding'. The main reason is that by unfolding in this way the whole experiment is considered as an object and it is possible to compare between experiments, i.e. the baseline for a healthy structure with subsequent states of the structure during operation (Nomikos and MacGregor 1994, Westerhuis et al 1999). The methodology for ' $D$ type unfolding' is illustrated in figure 2.

The physical variables present in most experiments do not have the same magnitudes and scales. It is necessary to treat the initial experimental data before any statistical analysis can take place. In the literature, there are a variety of techniques to rescale experimental data. For unfolded matrices, several scaling techniques have been studied. Among the most used techniques are continuous scaling (CS), variable scaling (VS), group scaling (GS), autoscaling (AS), etc. However, the most common method used for unfolded matrices is group scaling. The main reason is that group scaling considers the interaction between different sensors and processes them together. In general, all the methods are techniques that consist of rescaling each of the variables so that they have a mean of zero magnitude and the same variance or a variance equal to one (depending on the technique) (Westerhuis et al 1999, Kouti and MacGregor 1995, Gurden et al 2001, Villez et al 2009, Mujica et al 2010a, 2010b, Nomikos and MacGregor 1994, Wold et al 1998).

Once the matrix $X_{3 \mathrm{D}}$ has been unfolded into a new matrix $\bar{X}$, centered and scaled, a PCA study is performed based on the covariance matrix which quantifies the degree of linearity between all possible pairs of variables (in the remainder of the paper the bar notation over the $X$ means that the data have been scaled). Then, it is possible to order the eigenvectors associated with the covariance matrix in descending order (according to their associated eigenvalues); in this way, only a few principal eigenvectors can be selected; these represent the more important system dynamics. As result, an important dimensional reduction can be obtained.

The covariance matrix is given by

$$
C_{\bar{X}}=\frac{1}{n-1} \bar{X}^{\mathrm{T}} \bar{X} .
$$

The main diagonal terms of the covariance matrix represent the variances; therefore, the off-diagonal terms represent the covariances between pairs of variables,

$$
{\sigma_{\bar{v}}}_{j}^{2}=\frac{1}{n-1} \bar{v}_{j}^{\mathrm{T}} \bar{v}_{j}=\frac{1}{n-1} \sum_{i=1}^{n} \bar{x}_{i j}^{2} \text {, }
$$




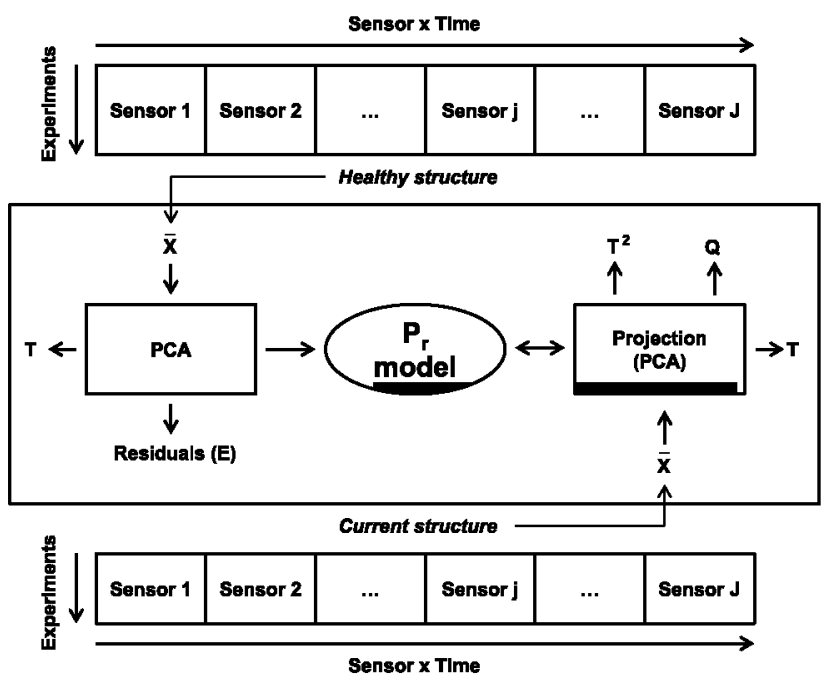

Figure 3. The scheme of the PCA model for structural damage identification.

$$
\sigma_{\bar{v}_{j}, \bar{v}_{k}}^{2}=\frac{1}{n-1} \bar{v}_{j}^{\mathrm{T}} \bar{v}_{k}=\frac{1}{n-1} \sum_{i=1}^{n} \bar{x}_{i j} \bar{x}_{i k} .
$$

Once the covariance matrix has been obtained, the data matrix $\bar{X}$ can be transformed using a linear transformation in order to achieve the minimal redundancy,

$$
T=\bar{X} P \text {. }
$$

This linear transformation must be such that the new data matrix $T$ is diagonal, i.e.,

$$
C_{T}=\frac{1}{n-1} P^{\mathrm{T}} \bar{X}^{\mathrm{T}} \bar{X} P=P^{\mathrm{T}} C_{\bar{X}} P .
$$

The transformation matrix is selected to have the eigenvectors by columns, i.e.,

$$
P=\left(p_{1} p_{2} \cdots p_{j} \cdots p_{m}\right) .
$$

Since eigenvectors are ordered according to the amount of information, the dimensionality of the data matrix $\bar{X}$ can be reduced if only a certain number $r$ of principal components is chosen,

$$
P_{r}=\left(p_{1} p_{2} \cdots p_{r}\right)
$$

The $T$ matrix (called the 'score matrix') has uncorrelated row vectors and its column vectors are the projections of the original data over the direction of the $j$ th principal component. These column vectors are called 'scores'.

A baseline must be constructed using data for the healthy structure. This means that the matrix $P_{r}$ must be calculated for the healthy structure. Later, results for an unknown structure condition $(\bar{X})$ should be projected into the baseline model,

$$
T=\bar{X} P_{r} .
$$

The methodology is outlined in figure 3.

From this projection, it is possible to calculate different damage indices and detection thresholds (Mujica et al 2010a, 2010b).
There are statistical metrics that, used along with PCA, allow detection of anomalous behavior in systems. These quantitative indices are intended to consider whether the results of different experiments or studies are homogeneous or not, provide information about the magnitude of the effect of the relationships studied together with a confidence interval and statistical significance and whether there is heterogeneity between different experiments or studies, and identify variables or characteristics that may affect the results. The two most common tools are the $Q$ index (or squared prediction error index) and the $T^{2}$ index (or $D$ index). The index $Q$ indicates how well each sample fits the PCA model. It is a measure of the difference between a sample and its projection in the main components retained by the PCA model (Jackson and Mudholkar 1979, Villez et al 2009, Burgos et al 2010).

The $T^{2}$ index is a measurement of the variation of each sample in the PCA model. It is based on analysis of the score matrix $(T)$ which allows study of the variability of the projected data in the new principal component space (Mujica et al 2010a, 2010b).

The $Q$ index is given by

$$
Q_{i}=\tilde{x}_{i} \tilde{x}_{i}^{\mathrm{T}}=x_{i}\left(I-P P^{\mathrm{T}}\right) x_{i}^{\mathrm{T}},
$$

where $\tilde{x}_{i}$ is the projection into the residual subspace.

The $T^{2}$ index is given by

$$
T_{i}^{2}=\sum_{j=1}^{r} \frac{t_{s i j}^{2}}{\lambda_{j}}=\frac{t_{s i} t_{s i}^{\mathrm{T}}}{\Lambda}=\frac{x_{i} P P^{\mathrm{T}} x_{i}^{\mathrm{T}}}{\Lambda} .
$$

\section{Experimental setup}

To validate the methodology, a $1.5 \mathrm{~m}$ wing section fully made in composite materials, belonging to an unmanned air vehicle, was used. Two fiber optics were bonded at the intrados and two at the extrados, each one having eight FBGs. In total, 32 FBGs were used. In addition, an optical fiber for distributed sensing was bonded along with optical fibers (FBGs) located at the leading edge in order to validate the strain measurements made with the FBGs. The wing was fixed to a testing bench by mean of screws in the same way as it is fixed to the fuselage on the aircraft. Once the wing was fixed, the testing phase began. The first step consisted in gathering the responses using the healthy structure to build the baseline PCA model. Each experiment consisted in loading the structure in the bending mode, with progressive loading from zero load to a specific load. After waiting $10 \mathrm{~s}$ for load stabilization, the load was removed progressively. The sampling rate was $10 \mathrm{~Hz}$. Four different loads were used in the bending mode $(3.25,4.75,6.25$ and $7.25 \mathrm{~kg})$, plus zero load. Each load case and the zero load case were repeated ten times to build the baseline model and ten times more for model validation. That is, additional data for the healthy structure were taken in order to validate the baseline model. Figure 4 illustrates the wing section used. The zero load cases were useful in order to verify that no residual stresses appeared after damage promotion. 


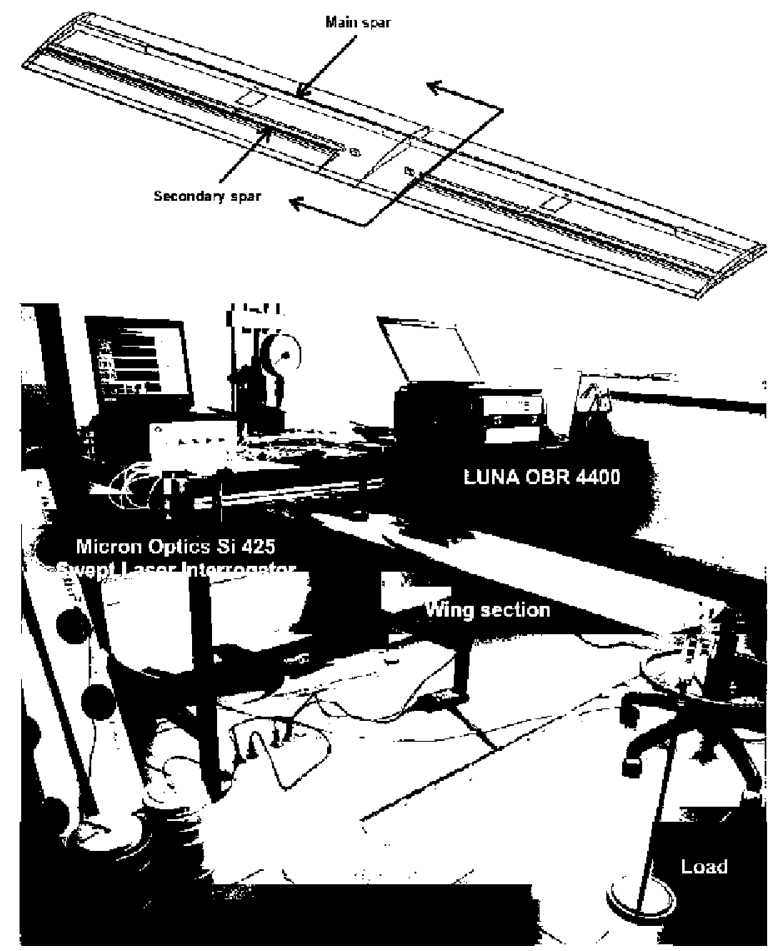

Figure 4. Top: scheme of the wing section used. Bottom: experimental setup.

A Micron Optics Si 425 interrogator was used for data acquisition. This equipment has a tunable laser and can interrogate up to 512 optic sensors in four channels at the same time at a maximum sampling rate of $250 \mathrm{~Hz}$ with a resolution of less than $0.2 \mathrm{pm}$. A Luna OBR 4400 (optical backscatter reflectometer) was used for distributed sensing.

After building the model for the healthy structure, two kinds of accumulative artificial damage were induced in the locations shown in figure 5. The first damage case consisted of a longitudinal skin cutting of $1 \mathrm{~cm}$. The second damage case consisted of increase of the size of the first crack to $3 \mathrm{~cm}$. The third damage case consisted of a transverse skin cutting of $1 \mathrm{~cm}$ without cutting the spar cap. From the fourth to the seventh cases, the transverse crack was increased by $1 \mathrm{~cm}$ each time and the spar cap was also superficially cut. Again, for each damage case, the zero load and the four different load cases were used and each experiment was performed ten times. In total, 400 experiments were performed, each one consisting of the signal of 32 sensors for more than 400 instants of time.

From the signal from each sensor, the initial zero load region and the stable load region (zones 1 and 2 respectively in figure 6) were isolated and preprocessed in order to remove outliers. The Ferguson test was used (kurtosis coefficient given by equation (12)) (ASTM E178 1972),

$$
b_{2}=\frac{n \sum_{i=1}^{n}\left(x_{i}-\bar{x}\right)^{4}}{(n-1)^{2} s^{4}}=\frac{n \sum_{i=1}^{n}\left(x_{i}-\bar{x}\right)^{4}}{\left[\sum_{i=1}^{n}\left(x_{i}-\bar{x}\right)^{2}\right]^{2}},
$$

where $s$ represents the standard deviation, $\bar{x}$ represents the arithmetic average and $n$ represents the number of samples.
To apply the test proposed by Ferguson, the $b_{2}$ value must be computed, and if the value exceeds the desired significance level, the observation farthest from the mean is rejected and the same procedure is repeated until no more values are judged to be outliers. In this experiment a significance level of $5 \%$ was selected.

After removing the outliers, the average of zone 1 in figure 6 was taken as reference (initial wavelength) and the average of zone 2 in the same figure, was taken as the final wavelength for strain calculation. In this way, since the experiments were performed in a room with a controlled temperature and each one took no more than $45 \mathrm{~s}$, thermal effects could be neglected. No wavelength shifting between zones 1 and 3 (figure 6) meant that no thermal effects and no residual stresses appeared during the experiments.

The strains for each experiment were calculated using

$$
\frac{\Delta \lambda_{\mathrm{B}}}{\lambda_{\mathrm{B}}}=\left(1-\rho_{\alpha}\right) \Delta \varepsilon+(1+\xi) \Delta T,
$$

where $\lambda_{\mathrm{B}}$ is the Bragg wavelength, $\rho_{\alpha}$ is the photoelastic coefficient of the fiber optics and $\varepsilon$ is the thermo-optic coefficient for the fiber optics.

For the same kind of fiber optics as used in this work, García obtained the following experimental constants (García 2010):

$$
\begin{aligned}
\Delta \varepsilon & =(803.9 \pm 5.6) \frac{\mu \varepsilon}{\mathrm{nm}}(\Delta \lambda) \rightarrow k_{\varepsilon} \\
& =(0.7991 \pm 0.0055) \mu \varepsilon^{-1}, \\
\Delta T & =(101.9 \pm 1.2) \frac{\mathrm{K}}{\mathrm{nm}}(\Delta \lambda) \rightarrow k_{T} \\
& =(6.334 \pm 0.074) \times 10^{-6} \mathrm{~K}^{-1},
\end{aligned}
$$

where $k_{\varepsilon}=1-\rho_{\alpha}$ and $k_{T}=1+\xi$.

Before proceeding to the application of PCA, the data were unfolded using the methodology schematized in figure 2 , centered and scaled using group scaling (GS). By means of group scaling each datum is scaled using the mean of all measurements of a sensor at the same instant of time and the standard deviation of all measurements of a sensor as follows (Kouti and MacGregor 1995, Sierra and Güemes 2011):

$$
\bar{x}_{i j k}=\frac{x_{i j k}-\mu_{j k}}{\sigma_{j}},
$$

where $x_{i j k}$ is the $k$ th sample of the $j$ th sensor in the $i$ th experiment, $\mu_{j k}$ is the mean of all $k$ th samples of the $j$ th sensor, $\mu_{j}$ is the mean of all measurements of the $j$ th sensor, $\sigma_{j}$ is the standard deviation of all measurements of the $j$ th sensor and $\bar{x}_{i j k}$ is the scaled sample.

$\mu_{j k}, \mu_{j}$ and $\sigma_{j}$ are given by

$$
\begin{gathered}
\mu_{j k}=\frac{1}{I} \sum_{i=1}^{I} x_{i j k}, \\
\sigma_{j}=\sqrt{\frac{1}{I K} \sum_{i=1}^{I} \sum_{k=1}^{K}\left(x_{i j k}-\mu_{j}\right)^{2},} \\
\mu_{j}=\frac{1}{I K} \sum_{i=1}^{I} \sum_{k=1}^{K} x_{i j k} .
\end{gathered}
$$



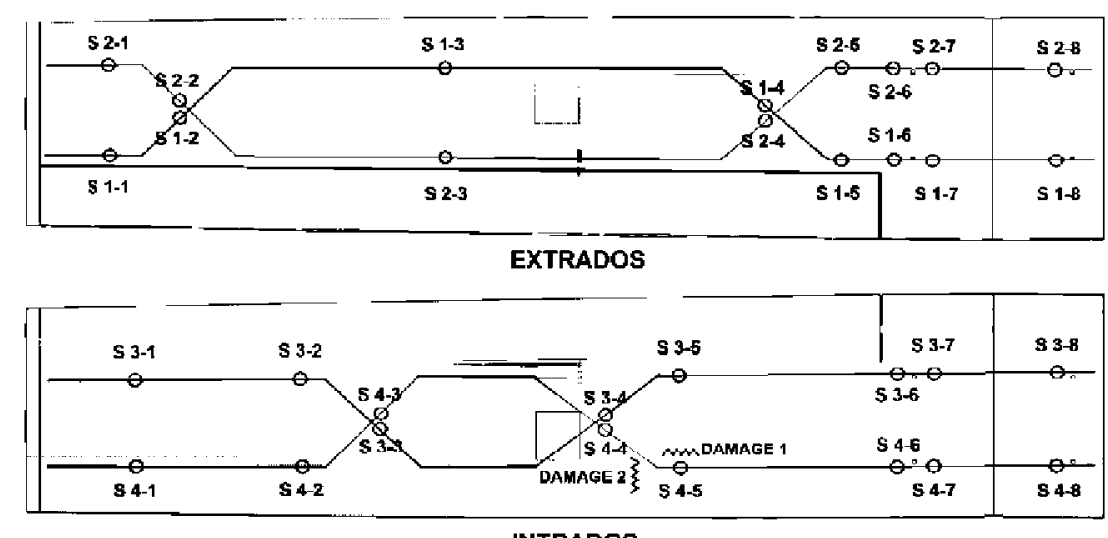

INTRADOS

Figure 5. The sensors and the induced damage locations.

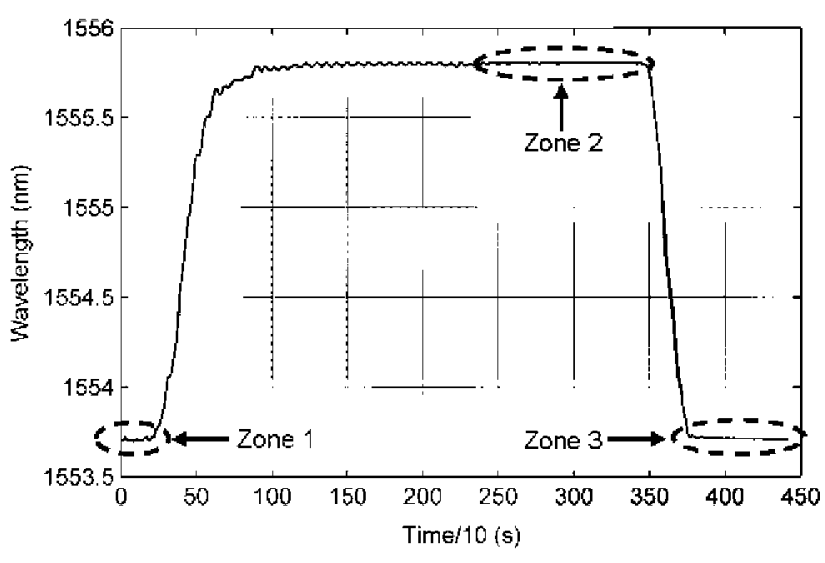

Figure 6. An example of the load spectrum and interest zones.

It should be remembered that $I$ represents the experiments, $K$ represents the samples and $J$ represents the sensors.

Finally, PCA was performed for the healthy structure and the different damage cases were projected onto the model. In this study, 20 principal components were selected to build the model.

\section{Analysis of the results}

In order to verify the baseline model, data for the healthy case were projected into the PCA model (for all the load cases). The additional data were not taken into account to build the PCA model. In this way, it was possible to compare between real damage cases and the healthy case, projecting the data into the model.

The projections in three planes (the $X-Y$ plane, the $Y-Z$ plane and the $X-Z$ plane) of the first three principal components are depicted in figure 7 . In the $X-Y$ plane, the projection into the first principal component versus the projection into the second principal component is presented. In the $Y-Z$ plane, the projection into the second principal component versus the projection into the third principal component is presented, and in the $X-Z$ plane, the projection into the first principal component versus the projection into the third principal component is presented. It is more complex

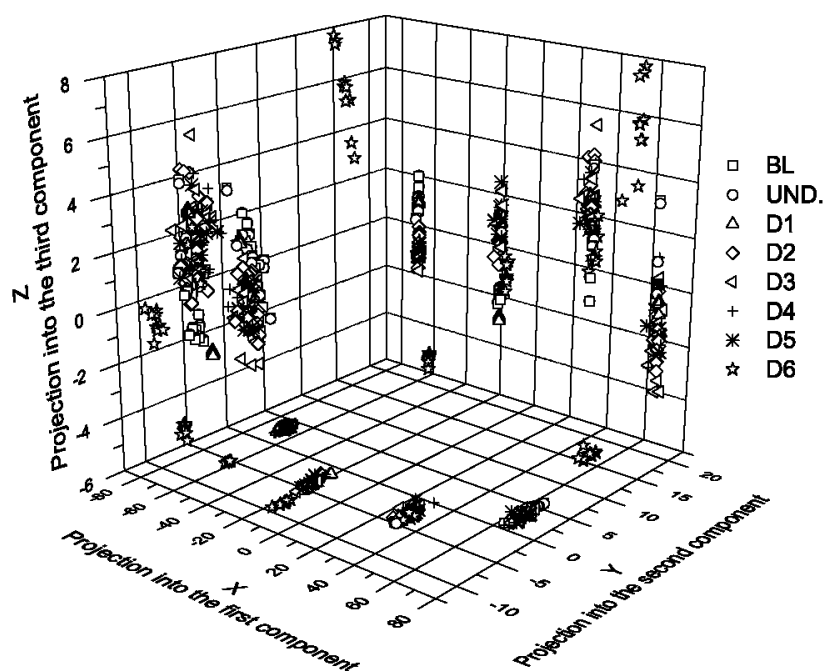

Figure 7. Projections into the first three principal components for all cases.

to try to represent more than three principal components in a single plot and it can be difficult to get valuable information from this.

Looking at figure 7 , it is possible to distinguish between four different segregated groups of data in each of the three planes. These data groups correspond to each of the various loading conditions studied. Inside each segregated group it is possible to distinguish tendencies for the different damage cases. If it is desired to obtain more precise information it is necessary to study each of these planes containing the different principal component projections. As Westerhuis et al showed, the sum of the variances of the two first principal components exceeds $80 \%$ of the original data variance for most cases (Westerhuis et al 1999). In this particular case, the two first principal components explain more than $95 \%$ of the variance.

As explained before, the first two principal components have the largest amount of representative information about the system variance. Figure 8 shows a detailed view of the projection into the first principal component versus the projection into the second principal component. Beside this, 


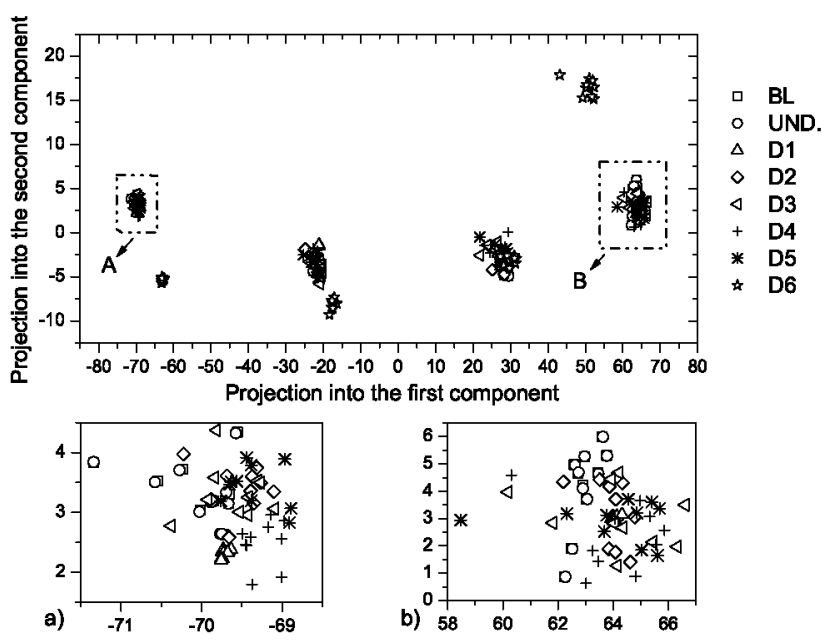

Figure 8. Projections into the first two principal components for all cases. (a) detail of zone 'A', (b) detail of zone 'B'.

two zoomed views are presented. The first zoom zone (marked with an ' $A$ ' in figure 8) corresponds to the lower load case and includes all the damage cases except the most severe one (represented by stars in the plot), for which the separation from the baseline is clear. The second zoom zone (marked with a ' $\mathrm{B}$ ' in figure 8) corresponds to the upper load case and, like in zoom ' $A$ ', includes all damage cases, except the most severe one.

In both zoomed views, a separation between the baseline and the different damage cases and a very good fit between the baseline and the undamaged case are evident.

Due to the appearance of nonlinear effects in the wing, under some load cases, the differences between the baseline and damage cases are not the same for the four studied load cases. In the third data group (at the left of zone 'B') for example, the data corresponding to the most severe damage case are closer to the other data than in the other three load cases.

Even though it is possible to see differences between the projections of the data associated with the load cases studied, it is necessary to integrate the results using quantitative indices that allow the results to be expressed in the same metric.

Figure 9 shows the $T^{2}$ index for all the different experiments, for all the load cases. Besides this, a zoomed area is presented below the figure (corresponding to the area marked with an 'A'). The zoomed area shows the load case for which, apparently, the indices for different damage cases are closer. However, looking closely, it is possible to appreciate that the baseline is the smallest one. All the indices associated with the damage are distant from the baseline and the indices associated with the undamaged case fit very well with the baseline.

Similarly, figure 10 shows the $Q$ index for all the experiments and all the load cases. A zoomed area is also presented below the figure, showing a detailed view of the same area as in figure 9. As in the previous analysis, in this region, corresponding to the third load case, all the data look very close to each other. However, on looking closely (excluding the data corresponding to the most severe damage

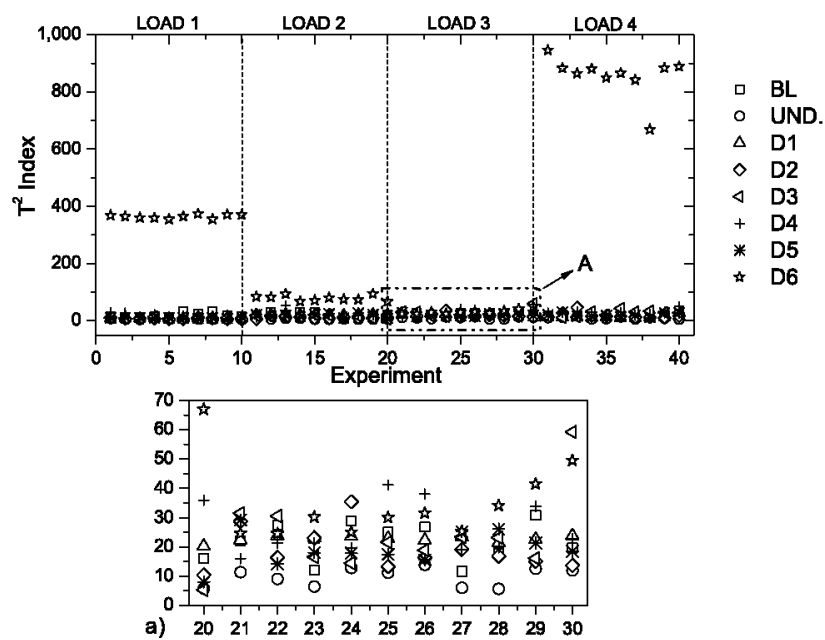

Figure 9. The $T^{2}$ index. (a) Detail of zone 'A'.

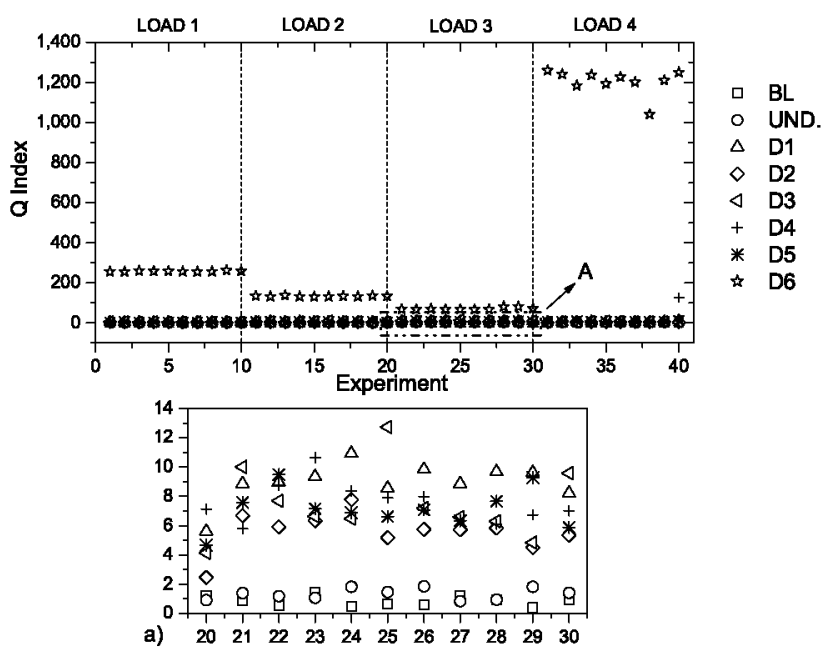

Figure 10. The $Q$ index. (a) Detail of zone 'A'.

case, for which there is an obvious separation from the baseline) it is possible to appreciate a separation between the indices for the different damage cases and the baseline. Besides, the indices associated with the undamaged case fit the baseline very well.

Comparing figures 9 and 10 it is possible to see a clearer tendency in figure 10 . All the damage cases can be clearly distinguished when are compared with the baseline. However, due to the nonlinear behavior of the wing section tested and the fact that several damage cases are mixed with several load cases all in one 'figure', it is very difficult to distinguish between the damage types in this figure.

In general, the $Q$ index is usually smaller than the $T^{2}$ index and any small change in the system characteristics can be observed. Because the $Q$ index explains how well a sample fits the PCA model, and is more sensitive than the $T^{2}$ index, the information obtained from this index can be considered more important in many cases. However, as it is a qualitative technique, one should beware of the inferences made.

Finally, in order to get more 'resolution' and obtain more accuracy in the diagnosis (or classification) of damage, a plot 


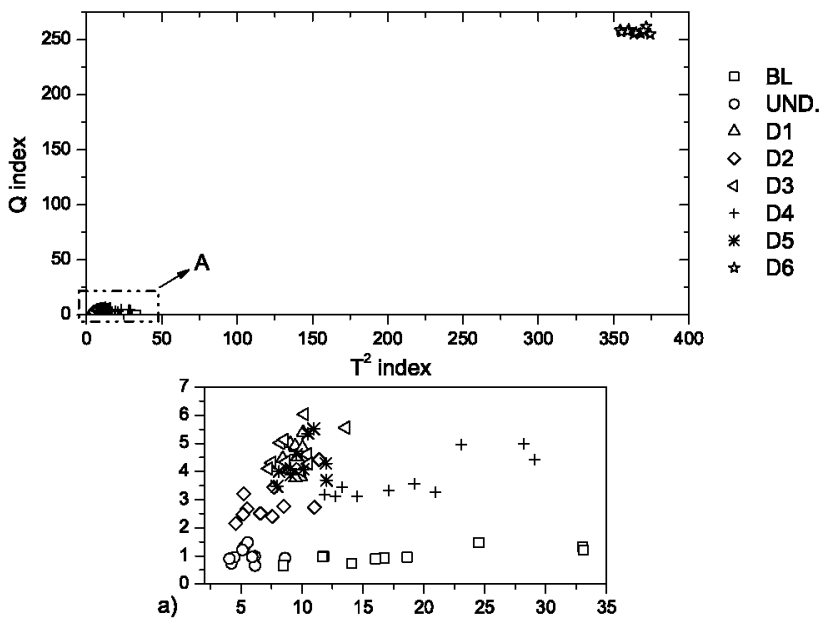

Figure 11. The $T^{2}$ index versus the $Q$ index for the lowest load case. (a) Detail of zone ' $\mathrm{A}$ '.

of the $T^{2}$ index versus the $Q$ index for the lowest load case is presented in figure 11. From this figure, it is very easy to see that the indices for the most severe damage case are away from the baseline. On the other hand, all the other damage cases look closer to the baseline, but this is due to the scale effect. On obtaining a closer view of the other damage cases by means of a zoomed view (presented below the figure and corresponding to the zone marked with 'A') it is possible to observe very clearly the independent groups associated with each damage case. In this particular case, there exists an overlapping between the third and fifth damage cases. Again, this is a consequence of the nonlinearities induced by the damage on the wing during the tests and the high strain levels achieved during some tests.

The $Q$ index is an error measurement. Because 'everything' is projected into a model (baseline), variations in the original data are more evident in the 'error' than in the projections.

In the same way, in figure 12, the $T^{2}$ index versus $Q$ index plot for the highest load case is presented. For this load case, the indices associated with the most severe damage case are also far away from the baseline. Again, zooming the area marked with 'A', it is possible to observe a separation between different damage cases and the baseline.

\section{Conclusions}

A PCA baseline model was built using the signals recorded by strain FBGs sensors during experiments with an undamaged structure. PCA modeling essentially consists of calculating the matrix P. During subsequent steps, experiments were performed using the structure in the different possible states (undamaged and six different damage cases). These signals were projected onto the PCA model, thus obtaining a selected number of first principal components ( $T$ scores). In addition, the $Q$ index and $T^{2}$ index were calculated. The approach has been experimentally analyzed showing good results in classifying different states of the structure: healthy structure and six different damage cases. The detection of damage

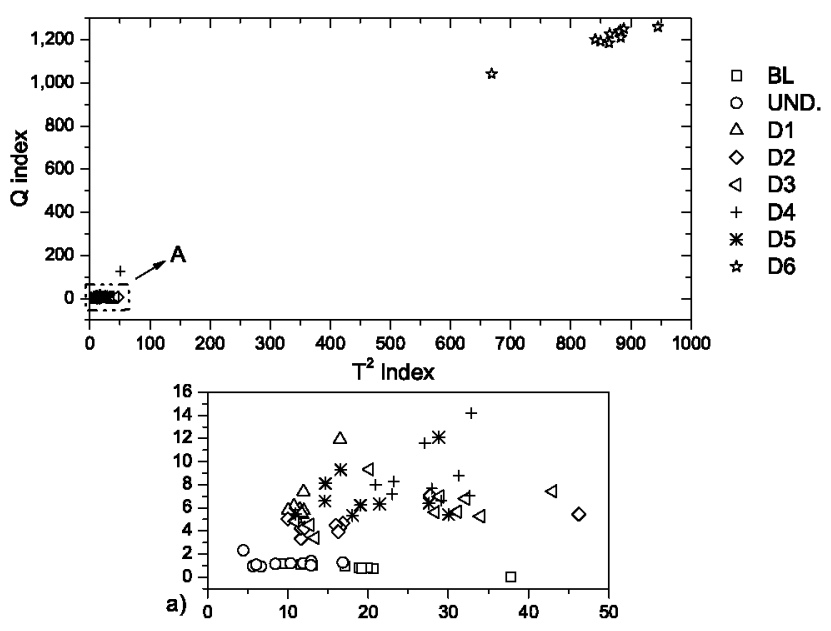

Figure 12. The $T^{2}$ index versus the $Q$ index for the highest load case. (a) Detail of zone ' $A$ '.

in complex structures using strain field pattern recognition by means of PCA and damage indices was possible. In general terms, it is possible to conclude that small defects slightly affect the global stiffness of the structure and those changes can be detected by mean of the proposed technique. Several experiments were carried out and analyzed using qualitative methods based on the $T$ scores, $T^{2}$ index and $Q$ index. In all cases it was possible to see deviations between the baseline and the data associated with the different damage cases. However, the $Q$ index shown be more sensitive in this particular study. It is necessary to develop strategies that allow a common conclusion to be reached from all the indices studied and, maybe, other indices available in the literature. Besides, it is necessary to develop thresholds that allow the severity of the damage to be classified quantitatively. The FBGs showed a very good sensitivity and were able to detect small strain changes in the structure under the same load conditions when small damages were induced. SHM models based on experimental data represent a robust methodology for damage detection. However, the use of these models in very complex structures has some limitations when the results are interpreted due the appearance of nonlinearities and other effects. In this work, several load condition were used in order to evidence the sensitivity of the technique. Due to the nonlinearities that appear under some load conditions with some induced damage, not only in the studied structure but also in other kinds of structures, it would be useful to conduct studies involving variable load conditions. Considering that each unfolding and scaling method studies a different kind of variability, new unfolding and scaling methods must be developed in order to avoid the need to study the results for the different load cases separately.

\section{Acknowledgments}

This work has been supported by the 'Ministerio de Ciencia e Innovación' in Spain through the coordinated research project DPI2011-28033-C03-03. 


\section{References}

Adams D 2007 Health Monitoring of Structural Materials and Components (Lafayette: Wiley) p 476

Balageas D, Fritzen C-P and Güemes A 2006 Structural Health Monitoring (London: ISTE-Hermes Science) p 496

Baseville M, Benveniste A, Goursat M and Mevel L 2007 IEEE Control Syst. Mag. 27 27-42

Burgos D, Mujica L, Güemes A and Rodellar J 2010 Active piezoelectric system using PCA 5th European Workshop on Structural Health Monitoring pp 164-9

Fritzen C-P and Kraemer P 2009 Mech. Syst. Signal Process. 23 1830-45

Fugate M, Sohn H and Farrar R 2001 Mech. Syst. Signal Process. $15707-23$

García C 2010 Aerospace Materials and Production Department Report (Madrid: Polytechnic University of Madrid)

Gurden S, Westerhuis J, Bro R and Smilde A 2001 Chemometr. Intell. Lab. Syst. 59 121-36

Holnicki-Szulc J 2008 Smart Technologies for Safety Engineering (Warsaw: Wiley) p 350

Jackson E and Mudholkar G 1979 Technometrics 21 341-9

Jolliffe I 2002 Principal Component Analysis (Aberdeen: Springer) p 487

Kerschen G and Golinval J 2004 Smart Mater. Struct. 13 211-9

Kouti T and MacGregor J 1995 Chemometr. Intell. Lab. Syst. $283-21$

Li D-S, Li H-N and Fritzen C-P 2012 Mech. Syst. Signal Process. $31217-27$

Lopez I and Sarigul-Klijn N 2010 Prog. Aerosp. Sci. 46 247-73

Mujica L E, Fernández A, Güemes A and Rodellar J 2010a Struct. Health Monit. 10 539-53
Mujica L, Tibaduiza D and Rodellar J 2010b Proc. Fifth World Conference on Structural Control and Monitoring (Shinjuku, Tokyo)

Mujica L, Vehí J, Staszewski W and Worden K 2006 Proc. Third European Workshop on Structural Health Monitoring (Granada) (Granada: DEStech Publications, Inc.) pp 601-8

Mujica L, Vehí J, Staszewski W and Worden K 2008 Struct. Health Monit. 7 215-30

Nair K and Kiremidjian A 2006 Proc. 4th World Conf. on Structural Control and Monitoring (San Diego)

Nomikos P and MacGregor J 1994 AIChE J. 40 1361-75

Ostachowicz W 2011 Proc. Int. Conf. New Trends in Structural Health Monitoring (Udine) (Udine: CISM) p 107

Reda M, Noureldin A, Lucero J and Baca T 2006 Struct. Health Monit. 5 267-95

Sierra J and Güemes A 2011 Proc. Comatcomp 2011 (Girona) pp 631-6

Sohn H, Czarnecki J and Farrar C 2000 J. Struct. Eng. 126 1356-63

Staszewski W 1997 Genet. Algorithms Eng. Syst.: Innov. Appl. $446186-90$

Staszewski W 2004 Health Monitoring of Aerospace Structures (Munich: John Wiley \& Sons) p 288

Villez K, Steppe K and De Pauw D 2009 Biosystems Eng. $10323-34$

Westerhuis J, Kourti T and MacGregor J 1999 J. Chemometr: $13397-413$

Wold S, Kettaneh N, Friden H and Holmberg A 1998 Chemometr. Intell. Lab. Syst. 44 331-40

Worden K and Dulieu-Barton J 2004 Struct. Health Monit. 3 85-98

Worden K and Farrar C 2007 Phil. Trans. R. Soc. Ser. A 365 303-15

Worden K and Manson G 2000 Inverse Probl. Eng. 8 25-46 